\title{
Método para calcular la eficiencia cuántica y la respuesta espectral de celdas solares usando LabVIEW
}

\section{Method to Calculate the Quantum Efficiency and Spectral Response of Solar Cells by Using LabVIEW}

\author{
Andrés Julián Aristizábal Cardona* \\ Departamento de Ingeniería. Universidad de Bogotá Jorge Tadeo Lozano. \\ Bogotá, Colombia
}

FECHA DE ENTREGA: 11 DE JUNIO DE 2015

FECHA DE EVALUACIÓN: 19 DE JUNIO DE 2015

FECHA DE APROBACIÓN: 23 DE JUNIO DE 2015

\begin{abstract}
Resumen En este trabajo se presentan los procedimientos clásicos para calcular la densidad de corriente espectral de cortocircuito para la base y para el emisor de una celda solar. Posteriormente, se calculan la eficiencia cuántica y la respuesta espectral. El método es realizado a partir de instrumentación virtual mediante el lenguaje de programación gráfica LabVIEW.

Este programa procesa los datos de longitud de onda, el coeficiente de absorción, el espectro de radiación y el coeficiente de reflexión para desarrollar el análisis mencionado y permite exportar a archivos en formato .xls, la información obtenida.

Los resultados indican para una celda solar de silicio; una densidad de corriente espectral de cortocircuito del emisor de $24,3 \mathrm{~mA} / \mathrm{cm}^{2} \mu$ (valor máximo) y de $61,6 \mathrm{~mA} / \mathrm{cm}^{2} \mu$ (valor máximo) para la base. La eficiencia cuántica interna alcanzó el $97 \%$ para los 0,65 $\mu \mathrm{m}$; mientras que la respuesta espectral interna registró $610 \mathrm{~mA} / \mathrm{W}$ en los $1,45 \mu \mathrm{m}$.
\end{abstract}

Abstract This article presents the classical procedures used to calculate the density of a short circuit spectral current for the base and the transmitter of a solar cell. Afterwards, we calculated its quantum efficiency

* Posdoctorado, Proyecto Distribución Eléctrica Inteligente SILICE, Universidad Nacional de Colombia, 2010. Doctorado en Ciencias - Física, Universidad Nacional de Colombia, 2008. Ingeniería Eléctrica, Universidad Nacional de Colombia, 2004. Director de la Maestría en Ingeniería - Gestión Sostenible de la Energía, Universidad de Bogotá Jorge Tadeo Lozano, 2014 - Actual. Áreas de interés: Energía Solar, Instrumentación Electrónica y Sensores, Calidad de Energía, Generación Distribuida. andresj.aristizabalc@utadeo.edu.co 
and spectral response. The method followed was virtual instrumentation through the graphic programming language LabVIEW.

The program developed processes wavelength data, the absorption coefficient, the radiation spectrum, and the reflection coefficient to carry out this analysis and export the information obtained to .xls files.

Results indicate that for a silicon solar cell and a short circuit, the spectral density current is $24.3 \mathrm{~mA} / \mathrm{cm}^{2} \mu$ (maximum value) with a base of 61.6 $\mathrm{mA} / \mathrm{cm}^{2} \mu$ (maximum value), the quantum efficiency reached $97 \%$ for $0.65 \mu \mathrm{m}$ and the spectral response registered $610 \mathrm{~mA} / \mathrm{W}$ at $1.45 \mu \mathrm{m}$.

Palabras Clave: celdas solares, eficiencia cuántica, respuesta espectral, densidad de corriente, instrumentación virtual.

Keywords: solar cells, quantum efficiency, spectral response, current density, virtual instrumentation.

\section{Introducción}

La tecnología fotovoltaica es una fuente de energía renovable prometedora que convierte la luz solar en electricidad, con un gran potencial para contribuir significativamente a solucionar el futuro problema energético que enfrenta la humanidad.

A la fecha, las celdas solares elaboradas a partir de materiales semiconductores dominan el mercado comercial, las de Si cristalino en un $80 \%$ de participación; el restante $20 \%$ es mayormente tecnología de películas delgadas, tales como CdTe y CIS y CIGS [1]. Las primeras son semiconductores de banda de energía prohibida o gap indirecta que normalmente requieren una capa de absorción de $300 \mathrm{~mm}$ de espesor y los costos de materiales y procesamiento son muy altos. Las segundas, contienen elementos que son tóxicos y de baja abundancia en la naturaleza. Sin embargo el compuesto $\mathrm{CuIn}_{12 \mathrm{x}} \mathrm{Ga}_{\mathrm{x}} \mathrm{Se}_{2}$ permite fabricar dispositivos solares de película delgada con mejor desempeño, ya que exhibe una eficiencia del $20 \%$, pero es 1,4 veces más costoso que los dispositivos de CdTe y de Si amorfo [2]. Una alternativa ecológica y de bajo costo para estos dispositivos de estado sólido, es la celda solar sensibilizada por colorante (DSC) [3] [4] y las celdas solares de materiales poliméricos que han alcanzado eficiencias cercanas al $9 \%$ (Solar Cell Efficiency Tables, Martin Green), cuyos costos de preparación son menores que los de las otras tecnologías dominantes en el mercado y su estructura permite la fabricación automatizada.

Las únicas celdas solares de silicio cristalino fabricadas por material de silicio grado solar a partir de un proceso metalúrgico, tienen un desempeño inferior que las fabricadas por silicio policristalino a partir de un proceso químico; pero las primeras tienen un voltaje de circuito abierto más alto que las últimas, en condiciones de poca iluminación [5].

El material de silicio grado solar a partir de un proceso metalúrgico, representa una manera rentable para la producción de celdas solares de silicio cristalino; pero es un sistema complejo de semiconductores: su calidad sufre por la presencia 
de impurezas de metales de transición, oxígeno, carbono y otras complejidades [6].

Los procesos de caracterización de celdas solares representan una fuente valiosa de información para mejorar los procesos ópticos y eléctricos de los materiales con el fin de aumentar el desempeño de los dispositivos fotovoltaicos, y de esta manera, lograr una disminución en los costos a nivel de producción industrial.

Los sistemas de instrumentación virtual son ampliamente usados en la industria y los laboratorios de enseñanza para experimentación, pruebas, control y análisis de desempeño de datos adquiridos mediante mediciones y para procesos de simulación y validación [7] [8] [9].

El proceso presentado en este trabajo, se efectuó mediante el paquete de software LabVIEW 2014, aplicando las definiciones clásicas de celdas solares sobre una celda solar de silicio caracterizada previamente; pero puede también ser utilizado para cualquier celda solar sin importar el tipo de tecnología, para la cual es necesario disponer de la información requerida por la aplicación desarrollada.

\section{Consideraciones iniciales}

\subsection{Modelo analítico de la celda solar}

El procedimiento de cálculo expuesto fue extraído del capítulo 2.2 de [10] y sus ecuaciones fueron procesadas mediante el software LabVIEW.

La celda solar es un dispositivo fabricado para convertir luz solar directamente en electricidad, mediante el fenómeno físico denominado efecto fotovoltaico [11]. Las celdas solares no necesitan recargarse como una batería, algunas han estado en operación continua a la intemperie en la tierra o en el espacio por más de 30 años [12].

La celda solar tiene la capacidad de absorber luz y entregar parte de la energía de los fotones absorbidos a los portadores de corriente eléctrica (electrones y huecos). Un semiconductor separa y colecta los portadores de carga y conduce la corriente eléctrica generada preferiblemente en una dirección específica. Así, una celda solar es simplemente un diodo semiconductor que ha sido diseñado y construido para absorber y convertir eficientemente la energía de la luz solar en energía eléctrica [13]. Una celda solar convencional se muestra en la figura 1 y su funcionamiento se explica a continuación.

La luz solar incide sobre la superficie de la celda solar. Una malla metálica hace contacto eléctrico con la celda y permite que la luz llegue al semiconductor por medio de la líneas de la malla y sea absorbida y convertida en energía eléctrica. Una capa anti reflectora entre las líneas de la malla, incrementa la cantidad de luz transmitida al semiconductor. Un diodo se forma cuando un semiconductor tipo $n$ y uno tipo $p$ son agrupados para formar una juntura. Esto se logra típicamente a través de procesos de difusión o implantación de impurezas específicas (dopantes) o mediante procesos de deposición. El otro contacto eléctrico del diodo es formado por una capa metálica en la parte posterior de la celda solar [12].

Los fenómenos para la generación de electricidad en la celda solar, se puede resumir en: absorción de radiación solar (absorción de fotones por parte de los 


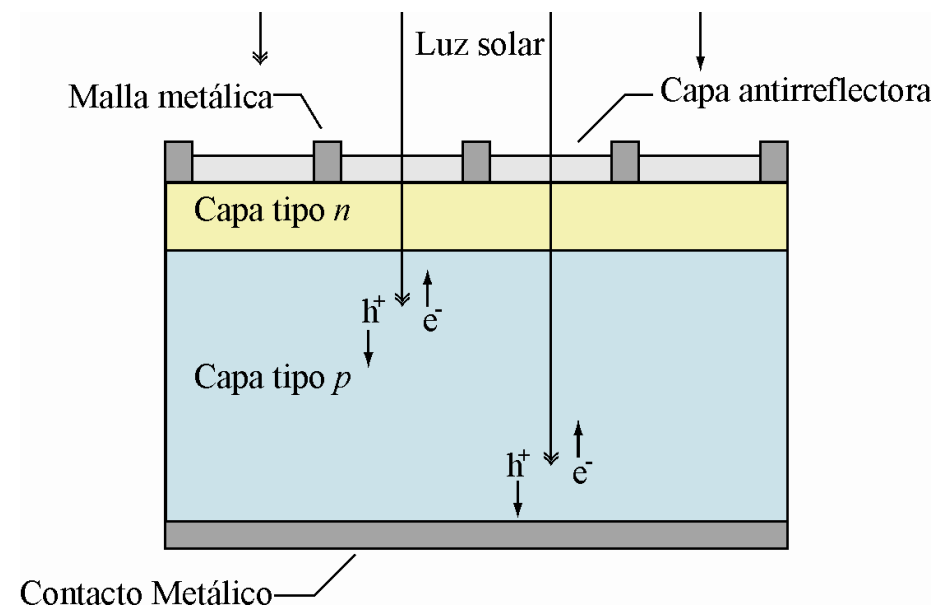

Figura 1. Representación esquemática de una celda solar convencional. Se representa la creación de pares electrón-hueco, $\mathrm{e}^{-} \mathrm{y} \mathrm{h}^{+}$, respectivamente [12].

electrones), separación de portadores de carga en la juntura, colección de esos portadores en los contactos del dispositivo y transporte hacia los diferentes tipos de acondicionamiento de potencia y almacenamiento de energía según el uso final determinado. Para aplicaciones terrestres, cada etapa de esta secuencia depende críticamente de las otras mencionadas. Por ejemplo, la generación fotovoltaica de electricidad debe ser acoplada con un proceso efectivo de almacenamiento con el fin de compensar la naturaleza intermitente de la radiación solar [14].

El cálculo de la corriente fotogenerada de una celda solar a una longitud de onda dada requiere la solución de un conjunto de cuatro ecuaciones diferenciales, incluyendo ecuaciones de continuidad y corriente tanto para portadores minoritarios como mayoritarios y la ecuación de Poisson [10].

\subsection{Densidad de corriente espectral de corto circuito}

Corresponde a la corriente por unidad de área y por unidad de longitud de onda incidente en la celda solar. El modelo descrito en [10] entrega el valor de la foto corriente recolectada por una superficie de celda solar de $1 \mathrm{~cm}^{2}$ cuando se expone a luz monocromática. Las expresiones analíticas son:

Densidad de corriente espectral de corto circuito del emisor:

$$
\begin{aligned}
J_{s c E}(\lambda) & =\frac{q \alpha \phi_{0}(1-R) L_{p}}{\left(\alpha L_{p}\right)^{2}}\left[-\alpha L_{p} e^{-\alpha W_{e}}\right. \\
& \left.+\frac{S_{e} \frac{L_{p}}{D_{p}}+\alpha L_{p}-e^{-\alpha W_{e}}\left(S_{e} \frac{L_{p}}{D_{p}} C h \frac{W_{e}}{L_{p}}+S h \frac{W_{e}}{L_{p}}\right)}{C h \frac{W_{e}}{L_{p}}+S_{e} \frac{L_{p}}{D_{p}} S h \frac{W_{e}}{L_{p}}}\right]
\end{aligned}
$$


Densidad de corriente espectral de corto circuito de la base:

$$
\begin{aligned}
J_{s c B}(\lambda) & =\frac{q \alpha \phi_{0}^{\prime}(1-R) L_{n}}{\left(\alpha L_{n}\right)^{2}-1}\left[-\alpha L_{n}\right. \\
& \left.-\frac{S_{b} \frac{L_{n}}{D_{n}}\left(C h \frac{W_{b}}{L_{n}}-e^{-\alpha W_{b}}\right)+S h \frac{W_{b}}{L_{n}}+\alpha L_{n} e^{-\alpha W_{b}}}{C h \frac{W_{b}}{L_{n}}+S_{b} \frac{L_{n}}{D_{n}} S h \frac{W_{b}}{L_{n}}}\right]
\end{aligned}
$$

Los principales parámetros de las ecuaciones (1) y (2) se definen en el cuadro 1 .

\begin{tabular}{cll}
\hline Símbolo Nombre & Unidades \\
\hline$\alpha$ & Coeficiente de absorción & $\mathrm{cm}^{-1}$ \\
$\phi_{0}$ & Flujo espectral de fotones en la superficie del emisor & Fotón $/ \mathrm{cm}^{2} \mu \mathrm{ms}$ \\
$\phi_{0}^{\prime}$ & Flujo espectral de fotones en la interface base - emisor & Fotón $/ \mathrm{cm}^{2} \mu \mathrm{ms}$ \\
$L_{n}$ & Longitud de difusión de electrones en la capa base & $\mathrm{cm}$ \\
$L_{p}$ & Longitud de difusión de los huecos en la capa del emisor & $\mathrm{cm}$ \\
$D_{n}$ & Constante de difusión de electrones en la capa base & $\mathrm{Cm}^{2} / \mathrm{s}$ \\
$D_{p}$ & Constante de difusión de huecos en la capa del emisor & $\mathrm{Cm}^{2} / \mathrm{s}$ \\
$S_{e}$ & Velocidad de recombinación en la superficie del emisor & $\mathrm{cm} / \mathrm{s}$ \\
$S_{p}$ & Velocidad de recombinación en la superficie de la base & $\mathrm{cm} / \mathrm{s}$ \\
$R$ & Coeficiente de reflexión & - \\
\hline
\end{tabular}

Cuadro 1. Principales parámetros involucrados en el modelo analítico [10].

\subsection{Flujo espectral de fotones}

El flujo espectral de fotones $\phi_{0}$ recibido en la superficie frontal del emisor de una celda solar, se relaciona fácilmente con la radiación espectral y la longitud de onda. Es importante aclarar que la radiación espectral es la potencia por unidad de área y unidad de longitud de onda. Lo anterior se muestra en la ecuación (3):

$$
\phi_{0}=10^{16} \frac{I_{\lambda} \lambda}{19.8}\left[\frac{\text { Fotón }}{\mathrm{cm}^{2} \mu \mathrm{ms}}\right]
$$

Con $I_{\lambda}\left(I\right.$ - radiación solar, $\lambda$ - longitud de onda) en $\mathrm{W} / \mathrm{m}^{2} \mu \mathrm{m}$ y $\lambda$ en $\mu \mathrm{m}$. Para la región de la base, se tiene la ecuación (4):

$$
\phi^{\prime}=\phi_{0} e^{-\alpha W_{e}}=e^{-\alpha W_{e}} 10^{16} \frac{I_{\lambda} \lambda}{19.8}\left[\frac{\text { Fotón }}{\mathrm{cm}^{2} \mu \mathrm{ms}}\right]
$$

De esta manera, la densidad de corriente espectral de corto circuito total (ecuación (5)) es la suma de las ecuaciones (1) y (2): 


$$
J_{s c \lambda}=J_{s c E}(\lambda)+J_{s c B}(\lambda)
$$

\subsection{Corriente total de corto circuito}

Corresponde a la contribución total de corriente a todas las longitudes de onda de la luz incidente en la celda solar. Esta se describe por medio de la ecuación (6):

$$
J_{s c}=\int_{0}^{\infty} J_{s c \lambda} d \lambda=\int_{0}^{\infty}\left(J_{s c E}(\lambda)+J_{s c B}(\lambda)\right) d \lambda
$$

\subsection{Eficiencia cuántica (QE)}

Corresponde al número de electrones producidos en el circuito externo a la celda solar por cada fotón del espectro incidente. La eficiencia cuántica externa e interna se definen empleando las ecuaciones (7) y (8):

$$
\begin{gathered}
I Q E=\frac{J_{s c \lambda}}{q \phi_{0}(1-R)} \\
E Q E=\frac{J_{s c \lambda}}{q \phi_{0}}
\end{gathered}
$$

\subsection{Respuesta espectral}

Se define como la relación entre la densidad de corriente espectral de corto circuito y la radiación espectral. También se define la respuesta espectral interna y la externa empleando las ecuaciones (9) y (10):

$$
\begin{gathered}
I S R=0.808(I Q E) \lambda \\
E S R=0.808(E Q E) \lambda
\end{gathered}
$$

\section{Resultados}

Con el fin de aplicar el modelo descrito, en LabVIEW, se desarrolló el instrumento virtual denominado "photoncell.vi" cuyo panel frontal se muestra en la figura 2. El programa cuenta con cinco menús de selección:

- Parámetros (Parameters).

- Corrientes de corto circuito (Short-circuit currents).

- Eficiencia cuántica interna (Internal quantum efficiency).

- Respuesta espectral interna (Internal spectral response).

- Característica IV (IV characteristic). 


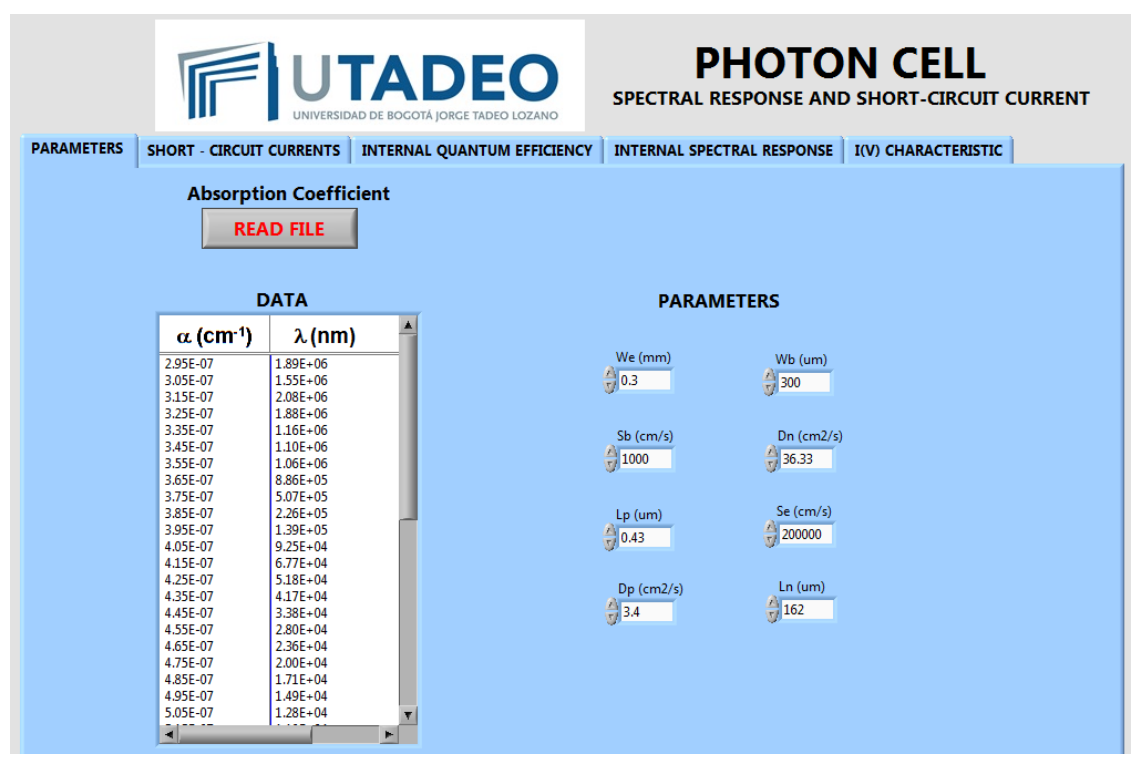

Figura 2. Panel frontal del programa "Photon Cell" desarrollado.

En el menú "Parámetros", el usuario debe inicialmente cargar el archivo que contenga los valores del espectro del coeficiente de absorción, en función de la longitud de onda de la celda solar a analizar. A continuación se deben ingresar los datos relacionados con el espesor y los demás parámetros indicados en el cuadro 1. El análisis se realizó considerando una celda solar de silicio con las dimensiones y características dadas a continuación [10]:

Espesor del emisor: $W_{e}=0,3 \mathrm{~mm}$, espesor de la base $W_{b}=300 \mu \mathrm{m}, L_{p}=$ $0,43 \mu \mathrm{m}, S_{e}=2 \times 105 \mathrm{~cm} / \mathrm{s}, D_{p}=3,4 \mathrm{~cm}^{2} / \mathrm{s}, L_{n}=162 \mu \mathrm{m}, S_{b}=1000 \mathrm{~cm} / \mathrm{s}$, $D_{n}=36,33 \mathrm{~cm}^{2} / \mathrm{s}$.

En la figura 3, se presentan las densidades de corriente espectral de corto circuito para la base y el emisor de la celda solar; así como también, la densidad de corriente total, las cuales fueron obtenidas a partir de los datos descritos en el párrafo anterior.

Se obtuvo una densidad de corriente espectral de cortocircuito del emisor de $24,3 \mathrm{~mA} / \mathrm{cm}^{2} \mu$ (valor máximo) y de $61,6 \mathrm{~mA} / \mathrm{cm}^{2} \mu$ (valor máximo) para la base.

El programa permite almacenar la información en archivos con formato .xls para realizar análisis posteriores, y crear un informe tipo html.

Como era de esperarse, la densidad de corriente espectral de cortocircuito en la base del dispositivo fotovoltaico es mayor respecto a aquella en el emisor; y más precisamente en $40 \mathrm{~mA} / \mathrm{cm}^{2}$ teniendo en cuenta que, en el emisor existen pérdidas en la generación de portadores asociados a los procesos de recombinación superficial del material. Tratamientos asociados al manejo anti reflectivo de la radiación solar en la capa del emisor, impactarían benéficamente la colección de portadores que permitirían aumentar la respuesta de la densidad de corriente total. En la figura 4, se puede apreciar la eficiencia cuántica interna de la celda solar. 


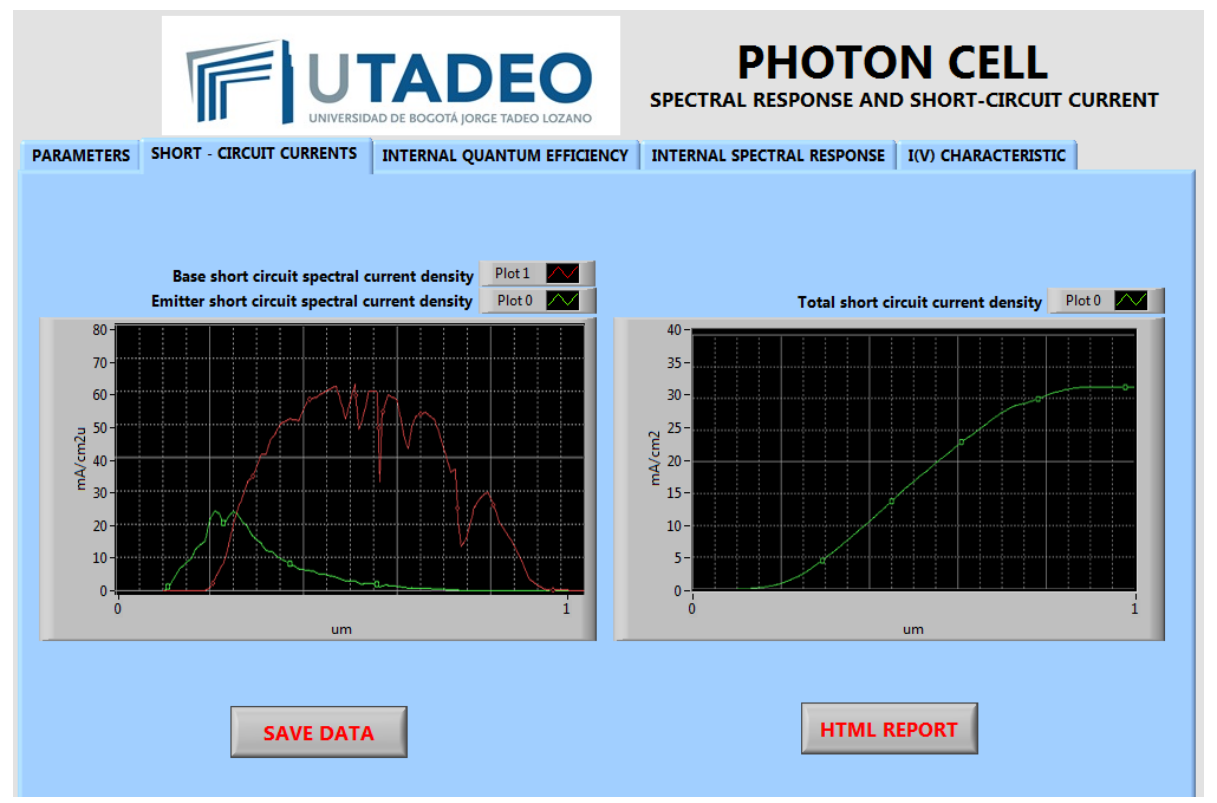

Figura 3. Densidades de corriente espectral de cortocircuito del emisor (verde - izquierda) y de la base (rojo - izquierda). Densidad total de corriente de cortocircuito (derecha).

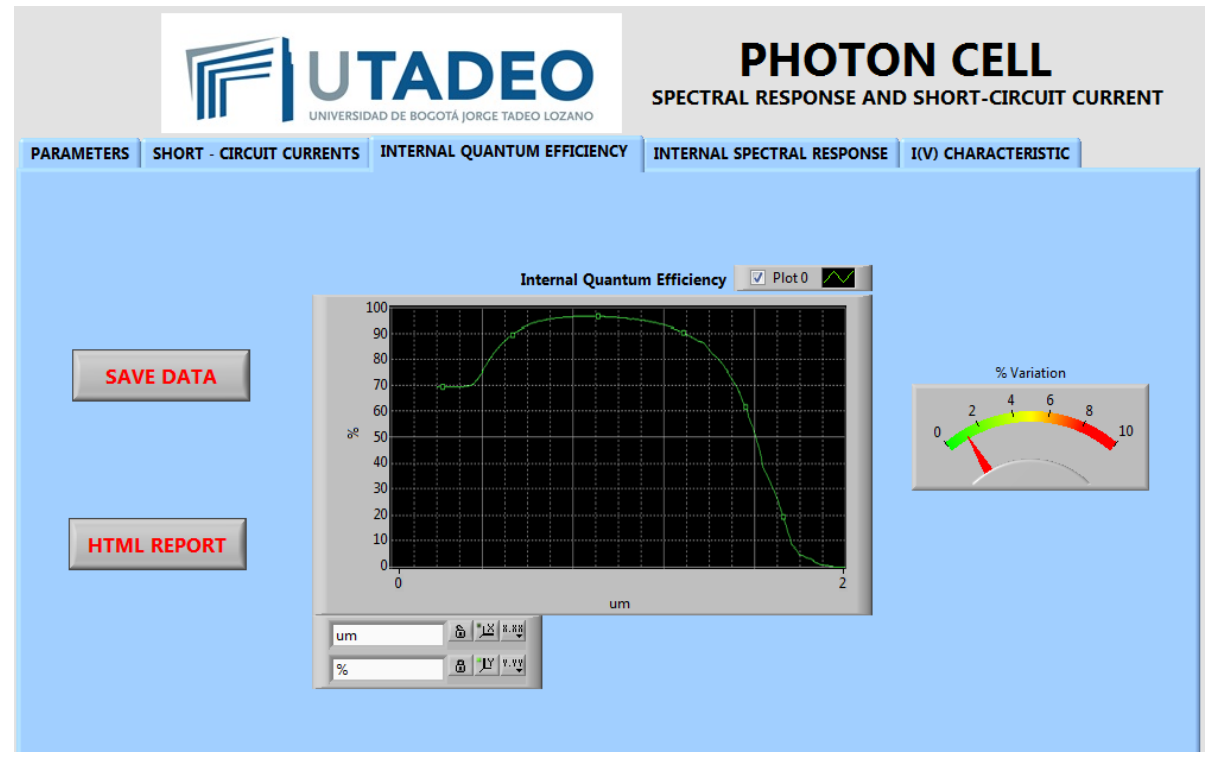

Figura 4. Respuesta de la eficiencia cuántica interna de la celda solar analizada.

La eficiencia cuántica interna alcanzó el $97 \%$ para los 0,65 $\mu \mathrm{m}$ de longitud de onda incidente. La eficiencia cuántica interna tiende a decrecer significativamente a partir de los 1,4 $\mu \mathrm{m}$ y ello puede deberse, al aumento de la luz reflejada por fotón incidente para cada longitud de onda a partir de dicho valor $(1,4 \mu \mathrm{m})$. 
De nuevo, la implementación de una capa anti reflectiva podría aumentar la cantidad de luz que llegue hasta la base y genere mayor cantidad de pares electrón - hueco y que los primeros puedan efectivamente hacer transición hacia la banda de conducción. En la figura 5 se muestra la respuesta espectral interna.

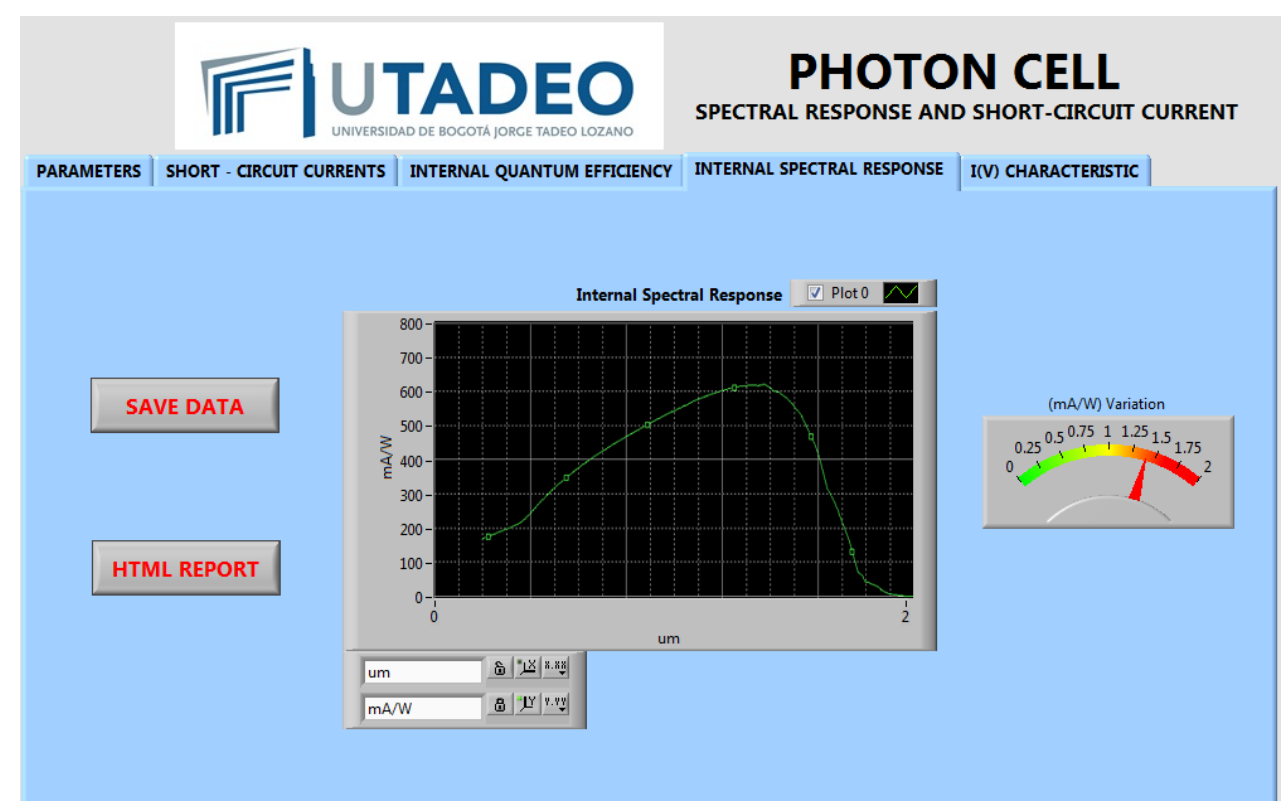

Figura 5. Respuesta espectral interna de la celda solar analizada.

La respuesta espectral interna registró un valor máximo de $610 \mathrm{~mA} / \mathrm{W}$ en los $1,45 \mu \mathrm{m}$. Se puede observar que para una longitud de onda de aproximadamente $0,30 \mu \mathrm{m}$, la celda registra una respuesta espectral interna cercana a $180 \mathrm{~mA} / \mathrm{W}$; mientras que para esa misma longitud de onda, la eficiencia cuántica interna era de alrededor del $70 \%$ (figura 4). Este comportamiento evidencia el inicio de la colección de portadores mayoritarios que va a definir hacia los $620 \mathrm{mV}$ el punto de máxima potencia del dispositivo (figura 5).

\subsection{Corriente en oscuro de la celda solar}

La curva I-V en oscuro de una celda solar es idéntica a la de un diodo convencional y se describe en la ecuación (11):

$$
I_{d}=I_{s}\left(e^{\frac{q V}{K T}}-1\right)
$$

\subsection{Efectos del material de la celda solar}

Tal como se mencionó anteriormente, varias magnitudes importantes en la conversión fotovoltaica dependen de las propiedades del material del semiconductor. 
En el cuadro 2, se presentan los principales parámetros de una celda solar de silicio y de una celda solar de GaAs [10].

\begin{tabular}{lll}
\hline Parámetro celda solar de silicio* & Celda solar de GaAs ${ }^{* *}$ \\
\hline$n_{i}\left(\mathrm{~cm}^{-3}\right)$ & $1 \times 10^{10}$ & $9,27 \times 10^{5}$ \\
$W_{e}(\mu \mathrm{m})$ & 0,3 & 0,2 \\
$L_{p}(\mu \mathrm{m})$ & 0,43 & 0,132 \\
$S_{e}(\mathrm{~cm} / \mathrm{s})$ & $2 \times 10^{5}$ & $5 \times 10^{3}$ \\
$D_{p}\left(\mathrm{~cm}^{2} / \mathrm{V}\right)$ & 3,4 & 7,67 \\
$W_{b}(\mu \mathrm{m})$ & 300 & 3,8 \\
$L_{n}(\mu \mathrm{m})$ & 162 & 1,51 \\
$S_{b}(\mathrm{~cm} / \mathrm{s})$ & $1 \times 10^{3}$ & $5 \times 10^{3}$ \\
$D_{n}\left(\mathrm{~cm}{ }^{2} / \mathrm{V}\right)$ & 36,63 & 46,8 \\
$J_{0}\left(\mathrm{~A} / \mathrm{cm}^{2}\right)$ & $1 \times 10^{-12}$ & $9,5 \times 10^{-20}$ \\
$J_{s c}\left(\mathrm{~A} / \mathrm{cm}^{2}\right)$ & $31,188 \times 10^{-3}$ & $25,53 \times 10^{-3}$ \\
\hline
\end{tabular}

Cuadro 2. Comparación entre una celda solar de silicio y una de GaAs. * Geometría de archivo PC1D5 Pvcell.prm, y cálculos usando ecuaciones en anexo 2 de [10]. ${ }^{* *}$ De L.D Partain, Solar Cells and their applications, Wiley 1995, p. 16.

\subsection{Superposición}

La superposición se emplea para determinar la corriente total de la celda solar al sumar las contribuciones bajo iluminación y oscuridad. Una vez han sido calculadas las corrientes de iluminación y bajo oscuridad de la celda solar, la corriente total es:

$$
J=J_{s c}-J_{\text {dark }}
$$

Sustituyendo la corriente bajo oscuridad se obtiene la ecuación (13):

$$
J=J_{s c}-J_{0}\left(e^{\frac{V}{V_{T}}}-1\right)
$$

\subsection{Característica I-V de una celda solar}

En este caso se usó "Photon Cell" aplicando la ecuación (13), para una celda solar con $1 \mathrm{~cm}^{2}$ de área, y los datos presentados en el cuadro $2 J_{s c}$ y $J_{0}$ para una celda solar de silicio. Simultáneamente, se calcula la característica I-V para una celda solar de GaAs usando la ecuación (13) y los datos del cuadro 2. El resultado de la curva I-V puede verse en la figura 6 .

Como puede observarse, comparando las características I-V para la celda solar de Si y para la de GaAs, los materiales diferentes de las celdas solares y 


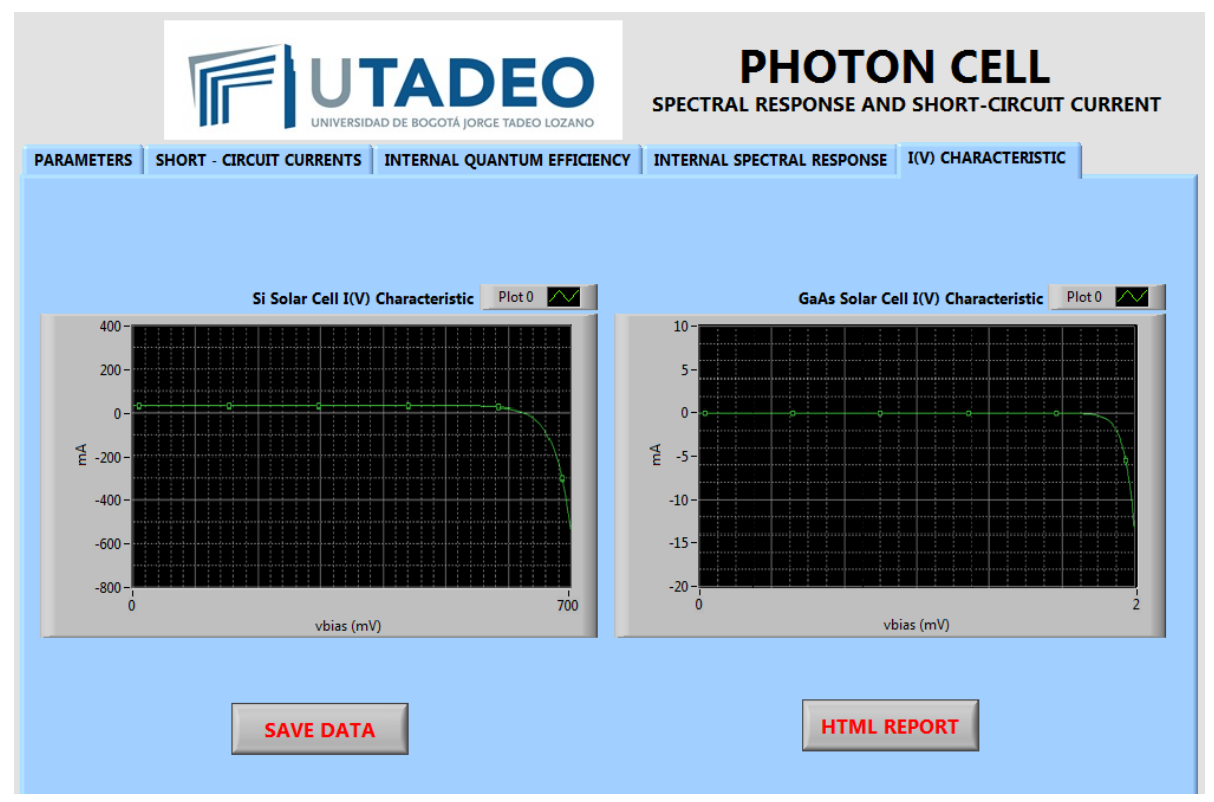

Figura 6. Cálculo de la característica I-V para una celda solar de Si y otra de GaAs.

sus geometrías producen diferentes niveles de corriente y voltaje. En particular puede notarse que la diferencia de voltaje es la más importante en este caso: el máximo valor de voltaje de la celda solar de GaAs es el doble que el de la celda solar de silicio, lo cual puede ser atribuido en mayor parte a la gran diferencia en los valores de $J_{0}[10]$.

\section{Conclusiones}

Se desarrolló una herramienta empleando instrumentación virtual que permite, previa medición del espectro de absorción, calcular los parámetros que caracterizan una celda solar.

Es posible representar el comportamiento de la eficiencia cuántica interna de una celda solar, con un modelo mono cristalino equivalente que considere parámetros efectivos.

La longitud de difusión es un parámetro relacionado directamente con la eficiencia cuántica interna de la celda solar y suministra información de los procesos de transporte, así como también del fenómeno de recombinación de portadores.

En el modelo empleado, se considera que la eficiencia de colección de portadores a lo largo de la región de carga espacial, se mantiene constante.

La eficiencia cuántica interna varía con la profundidad y puede ser calculada con parámetros gobernados exclusivamente por procesos de absorción.

Para el cálculo de la respuesta espectral, y en condiciones de corto circuito, la corriente es lineal con la irradiancia y es poco sensible frente a pequeños cambios 
de tensión, dependiendo directamente de la longitud de onda que incide sobre la celda solar.

Se verifica que a longitudes de onda pequeñas, la respuesta espectral de la celda solar decrece y se infiere que la energía mayor a la del gap del material, es efectivamente usada por la celda solar.

El cálculo de los parámetros descritos permite analizar otras propiedades de las celdas solares, útiles para caracterizar de forma integral el desempeño y eficiencia eléctrica de los dispositivos fotovoltaicos; así como también profundizar en aspectos que afectan los procesos de recombinación y el transporte eléctrico.

\section{Agradecimientos}

El presente documento ha sido soportado por el proyecto de investigación con código 633-11-14 financiado por la Universidad de Bogotá Jorge Tadeo Lozano.

\section{Referencias}

1. Bisquert, J.Dilemmasof. Dye sensitized solar cells. ChemPhysChem12, 1633-1636 (2011).

2. In Chung, Byunghong Lee, Jiaqing He, Robert P. H. Chang \& Mercouri G. Kanatzidis. All solid state dye sensitized solar cells with high efficiency. NATURE, VOL 485, MAY 2012.

3. Hagfeldt, A., Boschloo, G., Sun, L. C., Kloo, L. \& Pettersson, H. Dye-sensitized solar cells. Chem. Rev. 110, 6595-6663 (2010).

4. Gratzel, M. Recent advances in sensitized mesoscopic solar cells. Acc. Chem. Res. 42, 1788-1798 (2009).

5. Hong Yang, He Wang, Minqiang Wang. Investigation of open-circuit voltage in solar grade silicon solar cells from a metallurgical process route and cell's defect. Clean Techn. Environ. Policy (2013) 15:111-116.

6. Istratov AA, Buonassisi T, McDonald RJ et al (2003) Metal content of multicrystalline silicon for solar cells and its impact on minority carrier diffusion length. J. Appl Phys. 94:6552-6557.

7. B. Andó, S. Graziani and N. Pitrone, 'Stand-alone laboratory sessions in sensors and signal process- ing', IEEE Trans. Educ., 47 (2004), 4-9.

8. M. Nagistris, 'A Matlab-based virtual laboratory for teaching introductory quasistationary electro - magnetics', IEEE Trans. Educ., 48 (2005), 81-88.

9. T.W. Gedra, A. Seungwon, Q. H. Arsalan and S. Ray, 'Unified power engineering laboratory for electromechanical energy conversion, power electronics and power systems', IEEE Trans. Power Systems, 19 (2004), 112-119.

10. L. Castañer, S. Silvestre. 'Modelling Photovoltaic Systems Using PSpice'. Ed. Wiley, 2002.

11. Desarrollo de celdas solares basadas en $\mathrm{CuInSe}_{2}$ usando nuevos materiales buffer en su estructura. Clara Lilia Calderon Triana. Tesis de doctorado. Universidad Nacional de Colombia. 2003.

12. Handbook of Photovoltaic Science and Engineering. Edited by A. Luque and S. Hegedus. 2003 John Wiley \& Sons, Ltd ISBN: 0-471-49196-9. 
13. Síntesis y Caracterización de Nuevos Materiales No Tóxicos Empleados como Capa Buffer y Capa Absorbente en la Fabricación de Celdas Solares. Mónica Andrea Botero Londoño. Tesis de doctorado. Universidad Nacional de Colombia. Bogotá, 2008.

14. Fundamentals of solar cells. Photovoltaic Solar Energy Conversión. Alan L. Fahrenbruch and Richard H. Bibe. 1983. Academia Press, inc. ISBN: 0-12-247680-8. 